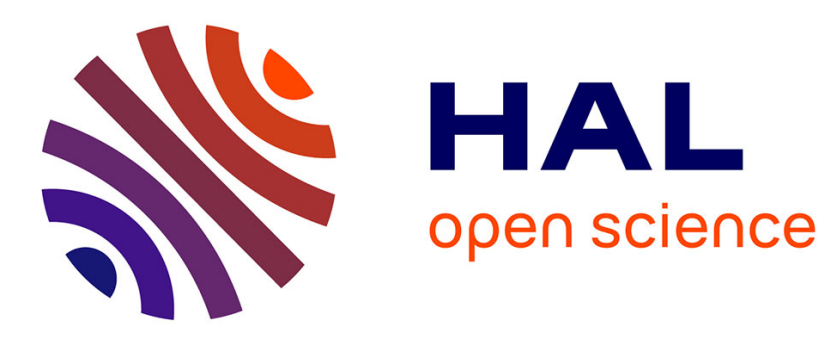

\title{
Reduced model for control in a hydroelectric unit at off-design operation
}

\author{
Simon Gerwig, Bilal Sari, Federica Garin, Carlos Canudas de Wit
}

\section{To cite this version:}

Simon Gerwig, Bilal Sari, Federica Garin, Carlos Canudas de Wit. Reduced model for control in a hydroelectric unit at off-design operation. ECC 2016 - 15th European Control Conference, Jun 2016, Aalborg, Denmark. pp.2096-2101. hal-01354358

\section{HAL Id: hal-01354358 \\ https://hal.inria.fr/hal-01354358}

Submitted on 30 Aug 2016

HAL is a multi-disciplinary open access archive for the deposit and dissemination of scientific research documents, whether they are published or not. The documents may come from teaching and research institutions in France or abroad, or from public or private research centers.
L'archive ouverte pluridisciplinaire HAL, est destinée au dépôt et à la diffusion de documents scientifiques de niveau recherche, publiés ou non, émanant des établissements d'enseignement et de recherche français ou étrangers, des laboratoires publics ou privés. 


\title{
Reduced Model for Control in a Hydroelectric Unit at Off-Design Operation
}

\author{
Simon Gerwig, Bilal Sari, Federica Garin and Carlos Canudas-de-Wit
}

\begin{abstract}
This paper describes the development of a reduced-order linear mathematical model of a hydroelectric unit subject to pressure perturbations in the draft tube of the hydraulic turbine, as it is the case at off-design operation (partial load or full load). The objective behind this development is to design a control algorithm able to attenuate the effect of these hydraulic pressure oscillations on the hydroelectric unit. First, we develop the reduced-order linear hydraulic model of the turbine and the pipes. Then this hydraulic model is combined with a reduced order model of a single machine infinite bus (SMIB) system. Finally we present simulation results of a controller designed with this model.
\end{abstract}

\section{INTRODUCTION}

Hydroelectric power plants contribute to the stability of the electrical network thanks to their fast time response in comparison to other electricity sources. The hydraulic turbine converts the potential energy of water stored in natural or artificial reservoirs into rotating mechanical energy, which is then converted to electricity by the electric generator. Fig. 1 is a drawing of a two-units hydroelectric powerplant showing two reaction type turbines with their draft tubes and the generators.

Typical Francis or pump turbines are designed for an optimal flow and are operated around this point. With the increase use of intermittent energy sources such as solar panels and wind turbines, the operating range of the turbines is extended. At off-design operation of the hydraulic turbine (when the flow through the turbine is smaller or larger than the optimal flow), some hydraulic phenomena set up in the draft tube and produce pressure oscillations. At partial load, for example, a helical vortex rope builds up in the draft tube and induces pressure oscillations in the hydraulic system with a frequency in the range of 0.2 to 0.4 times the turbine rotational frequency [1]. These pressure oscillations are converted into mechanical torque oscillations and eventually into electric power oscillations. Therefore the idea is to design a control algorithm able to attenuate the effect of these pressure oscillations on the hydroelectric unit. An important effort towards this aim is to find a suitable model of the physical system, simple enough to make the control design effective, but rich enough to capture the system behavior.

$\mathrm{S}$. Gerwig is a $\mathrm{PhD}$ candidate in the $\mathrm{NeCS}$ team, Inria, and at Alstom Hydro, France (email: simon.gerwig@inria.fr).

B. Sari is research engineer at Alstom Hydro, Process Control R\&D, Grenoble, France (email: bilal.sari@ power.alstom.com)

F. Garin is a researcher with the NeCS team, Inria, Grenoble, France (email: federica.garin@inria.fr)

C. Canudas-de-Wit is Director of Research at CNRS, GIPSA-Lab (NeCS team), Grenoble, France (email: carlos.canudas-de-wit@gipsa-lab.fr).

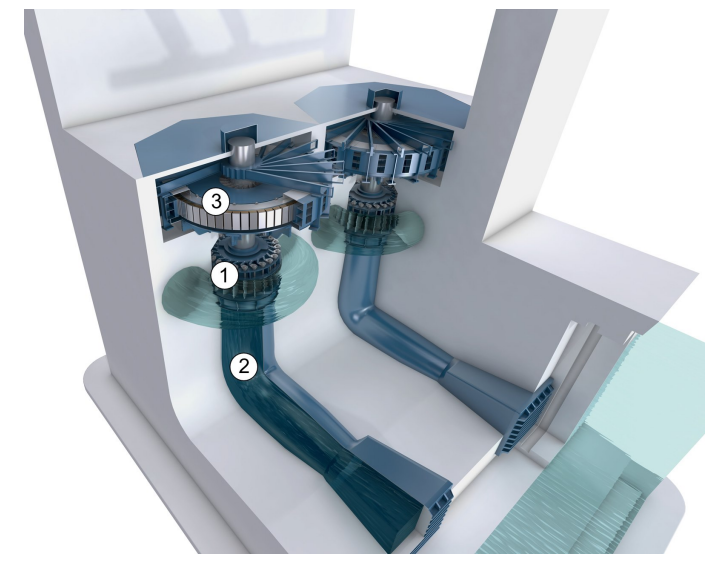

Fig. 1. Hydroelectric power plant picture with 1) the hydraulic turbine, 2) the turbine draft tube and 3) the synchronous generator.

\section{A. State of the Art}

The primary focus of the control literature of hydraulic turbine is to tackle the two main features of the hydraulic system, the non-minium phase characteristic and the nonlinearities of the turbine [2] [3]. Therefore mathematical models have been developed to represent these two aspects and [4] is the result of a working group on turbine modeling, where the authors develop several models of nonlinear ideal turbines along with models of pipes taking into account traveling waves or not through the use of hyperbolic functions.

Several strategies have been developed to design a controller able to control the nonlinear turbine, such as PID gain scheduling [5] which uses linearized characteristic curves for the turbine model, robust control [6] with the linear ideal model described in [4] and adaptive control [7].

Predictive control has been developed on a six-units power plant in [8] where the authors have developed a linear model of the hydraulic turbines sharing a common penstock and a linear model of the electric generator. They compare their proposed algorithm with the classical PI controller in terms of cross-coupling between the units.

One-dimensional dynamic models of pressure perturbations occurring at partial or full load exist in the hydraulic literature [9], [10] which rely on two parameters characterizing the vortices. A more simpler approach is to represent the pressure oscillations as an external perturbation as in [1]. The latter will be used here.

Some authors have considered the partial vortex effects on the active power. In [11], they consider torque oscillations at partial load and tune the power system stabilizer (PSS) 
of a the synchronous machine to damp the active power oscillations. In a similar manner, the author in [12] tunes the PSS of the unit to damp the effect of the vortex rope oscillations considering that there is an external perturbation acting on the mechanical torque.

\section{B. Paper Contribution}

In this paper, we develop a reduced order mathematical model of a hydroelectric unit subject to off-design pressure oscillations. We consider that an external perturbation is entering the system in the draft tube of the turbine, with the perturbation modeled as a sine function of known frequency. The hydraulic sub-system is represented by a linear model taking into account travelling pressure waves into the pipes using the finite elements method and the turbine is modeled by a linearization of its characteristic curves. After an order reduction, this hydraulic model is combined with a linear third order model of a synchronous generator connected to an infinite bus. We finally present simulation results of a controller designed with this model.

\section{Notations AND Definitions}

TABLE I

HYDRAULIC NOTATIONS

\begin{tabular}{r|l}
\hline$q(x, t)$ & Water flow in pipe at section $x$ and time $t\left(\mathrm{~m}^{3} / \mathrm{s}\right)$ \\
$q_{t}$ & Water flow through the turbine $\left(\mathrm{m}^{3} / \mathrm{s}\right)$ \\
$h(x, t)$ & Water head at section $x$ and time $t(\mathrm{~m})$ \\
$h_{n}$ & Water head difference between inlet and outlet of the turbine $(\mathrm{m})$ \\
$\alpha$ & Guide vane opening angle (degrees) \\
$T_{m}$ & Mechanical torque produced by the turbine \\
$x$ & Linear abscissa along the pipe $(\mathrm{m})$ \\
$t$ & Time $(\mathrm{s})$ \\
$g$ & Acceleration of gravity $=9.81 \mathrm{~m} / \mathrm{s}^{2}$ \\
$L$ & Pipe length $(\mathrm{m})$ \\
$A$ & Pipe area $\left(\mathrm{m}^{2}\right)$ \\
$D$ & Pipe diameter $(\mathrm{m})$ \\
$a$ & Wave propagation velocity $=1200 \mathrm{~m} / \mathrm{s}$ \\
$f$ & Head loss constant \\
\hline
\end{tabular}

\section{System Description And MathematicAl Model}

\section{A. System Physical Description}

We consider a hydroelectric unit where a penstock is providing water from an upstream reservoir to a reaction turbine (Francis for example), from which the turbine produces mechanical torque. This mechanical energy is then converted to electricity by a synchronous generator connected to the electrical network.

Mathematical dynamic models for off-design pressure oscillations in the draft tube have been developed in the hydraulic literature [10]. While these models are useful to understand the phenomena, they are too complicated for control design purposes. This is the reason why we consider a simpler approach where the pressure oscillations induced by off-design hydraulic phenomena are considered to be an external perturbation. This external perturbation $h_{w}$ will be mathematically represented by a sine function:

$$
h_{w}=A_{h} \sin \omega_{h} t
$$

TABLE II

ELECTRO-MECHANICAL NOTATIONS

\begin{tabular}{r|l}
\hline$P_{t}, Q_{t}$ & Active and reactive powers transmitted \\
$E_{t}, I_{t}$ & to the grid at machine terminals (p.u.) \\
$E_{f d}$ & Excitation voltage (p.u.) \\
$\Phi$ & Power factor angle (rad) \\
$\delta_{i}$ & Internal angle (rad) \\
$\omega_{r}$ & Rotational speed (p.u.) \\
$K_{D}$ & Friction coefficient (p.u.) \\
$H$ & Inertia constant (p.u.) \\
$e_{d}, e_{q}$ & Terminal voltage d, q-axis components (p.u.) \\
$i_{d}, i_{q}$ & Terminal current d, q-axis components (p.u.) \\
$E_{B d}, E_{B q}$ & Bus voltage d, q-axis components (p.u.) \\
$\delta$ & Load angle (rad) \\
$E_{B}$ & Bus voltage (p.u.) \\
$i_{f d}$ & Rotor field current (p.u.) \\
$\Psi_{a d}, \Psi_{a q}$ & d, q-axis flux linkage (p.u.) \\
$X_{d s}, X_{q s}$ & d, q-axis saturated reactances (p.u.) \\
$R_{a}$ & Stator resistance (p.u.) \\
$L_{a d u}, L_{a d s}$ & Unsat. and saturated mutual inductance in d-axis (p.u.) \\
$X_{E}, R_{E}$ & Line reactance and resistance (p.u.) \\
$L_{f d}, R_{f d}$ & Rotor field leakage inductance (p.u.) and resistance \\
$L_{l}$ & Leakage inductance (p.u.) \\
$X_{d s}^{\prime}$ & Transient d-axis saturated reactance (p.u.) \\
\hline
\end{tabular}

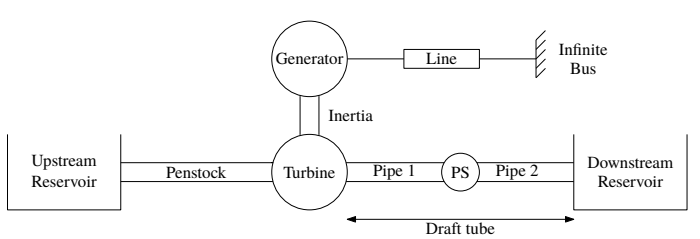

Fig. 2. Hydroelectric system schematic diagram. The hydraulic subsystem consists of an upstream reservoir, a penstock, a pump turbine which draft tube is modeled by two pipes in series with a pressure source (PS), and a downstream reservoir. The turbine provides rotating mechanical energy to the synchronous generator which is connected to the electrical grid through a line impedance.

where $A_{h}$ is the amplitude of the perturbation in meters, $\omega_{h}$ its frequency in $\mathrm{rad} / \mathrm{s}$ and $t$ the time in seconds. With laboratory and on-site measurements, it is possible to determine $\omega_{h}$. The amplitude $A_{h}$ is difficult to determine because this model of the perturbation is not directly measurable in reality and because it depends on the operation point, it should be treated as an unknown parameter.

\section{B. Hydraulic Subsystem Mathematical Model}

Turbine: The hydraulic turbine mathematical model is made of characteristic curves [2]. These charts are built from laboratory measurements and consist of nonlinear mappings:

$$
\begin{aligned}
q_{t} & =f_{1}\left(h_{n}, \omega_{r}, \alpha\right) \\
T_{m} & =f_{2}\left(h_{n}, \omega_{r}, \alpha\right) .
\end{aligned}
$$

Where $q_{t}, h_{n}, \omega_{r}, \alpha$ and $T_{m}$ are given in Table I and Table II. We can linearize these equations around an operating point $\left(q_{t_{0}}, h_{n_{0}}, \omega_{r_{0}}, \alpha_{0}, t_{m_{0}}\right)$ and we get

$$
\begin{aligned}
\Delta q_{t} & =\alpha_{1} \Delta h_{n}+\alpha_{2} \Delta \omega_{r}+\alpha_{3} \Delta \alpha \\
\Delta T_{m} & =\beta_{1} \Delta h_{n}+\beta_{2} \Delta \omega_{r}+\beta_{3} \Delta \alpha,
\end{aligned}
$$

where the $\alpha_{i}, \beta_{i}$ are the tangents of curve at the considered operating point. 
Pipes: The dynamics of the conduits is described by the hyperbolic partial differential equations:

$$
\begin{aligned}
a^{2} \frac{\partial q(x, t)}{\partial x}+g A \frac{\partial h(x, t)}{\partial t} & =0 \\
\frac{\partial q(x, t)}{\partial t}+g A \frac{\partial h(x, t)}{\partial x}+\frac{f}{2 D A} q(x, t)|q(x, t)| & =0 .
\end{aligned}
$$

We discretize these equations by using the finite elements method, we now consider that the pipe of length $L$ is made of $N_{b}$ pipe elements of length $\mathrm{d} x=L / N_{b}$. The number of elements $N_{b}$ has to be arbitrarily high to be as close as possible to reality. We can apply (4) on each small element and linearize around the operating point $\left(h_{0}, q_{0}\right)$. For $i=$ $1, \ldots, N_{b}$ we obtain:

$$
\begin{aligned}
\Delta q_{i+1}-\Delta q_{i}+\sigma_{1} \frac{\mathrm{d} \Delta h_{i+1 / 2}}{\mathrm{~d} t} & =0 \\
\Delta h_{i+1 / 2}-\Delta h_{i-1+1 / 2}+\sigma_{2} \frac{\mathrm{d} \Delta q_{i}}{\mathrm{~d} t}+\sigma_{3} \Delta q_{i} & =0
\end{aligned}
$$

where

$$
\sigma_{1}=\frac{A g \mathrm{~d} x}{a^{2}}, \quad \sigma_{2}=\frac{\mathrm{d} x}{A g}, \quad \sigma_{3}=\frac{f\left|q_{0}\right| \mathrm{d} x}{2 g D A^{2}} .
$$

Finally, the full model of a pipe discretized in $N_{b}$ elements can be expressed by

$$
\Lambda \frac{\mathrm{d}}{\mathrm{d} t}\left(\begin{array}{c}
\Delta q_{1} \\
\Delta h_{1+1 / 2} \\
\Delta q_{2} \\
\vdots \\
\Delta q_{N_{b}} \\
\Delta h_{N_{b}+1 / 2} \\
\Delta q_{N_{b}+1}
\end{array}\right)+\Sigma\left(\begin{array}{c}
\Delta q_{1} \\
\Delta h_{1+1 / 2} \\
\Delta q_{2} \\
\vdots \\
\Delta q_{N_{b}} \\
\Delta h_{N_{b}+1 / 2} \\
\Delta q_{N_{b}+1}
\end{array}\right)=\left(\begin{array}{c}
\Delta h_{\text {in }} \\
0 \\
0 \\
\vdots \\
0 \\
0 \\
-\Delta h_{\text {out }}
\end{array}\right),
$$

where $\Lambda$ and $\Sigma$ are defined by

$$
\begin{gathered}
\Lambda=\operatorname{diag}\left(\frac{1}{2} \sigma_{2}, \sigma_{1}, \sigma_{2}, \ldots, \sigma_{2}, \sigma_{1}, \frac{1}{2} \sigma_{2}\right) \\
\Sigma=\left(\begin{array}{ccccccc}
\frac{1}{2} \sigma_{3} & 1 & 0 & & & & \\
-1 & 0 & 1 & 0 & & & \\
0 & -1 & \sigma_{3} & 1 & 0 & & \\
& & & \ddots & & & \\
& & 0 & -1 & \sigma_{3} & 1 & 0 \\
& & & 0 & -1 & 0 & 1 \\
& & & & 0 & -1 & \frac{1}{2} \sigma_{3}
\end{array}\right) .
\end{gathered}
$$

Full reduced-order hydraulic subsystem: We use 40 elements for each pipe of the hydraulic system using (7) to have a good accuracy for the low frequency eigenvalues, and combine these equations with the turbine equations (2). This high order nonlinear model is implemented in the software package Simsen [13] and will be used later for the numerical simulations.

This model is linearized around the operating point $q_{0}=$ 0.5 p.u. where we observe the partial vortex rope. The head deviations at the upstream and downstream reservoirs are neglected and the external perturbation adds a difference in head between the outlet and the inlet of pipe 1 and pipe 2 respectively, see Fig. 2.
TABLE III

PARAMETERS OF THE CONSIDERED SYSTEM

\begin{tabular}{r|c}
\hline Rated active power & $130 \mathrm{MW}$ \\
Rotational speed & $136.36 \mathrm{rpm}$ \\
Rated head & $71 \mathrm{~m}$ \\
Rated flow & $200 \mathrm{~m}^{3} / \mathrm{s}$ \\
Penstock length & $400 \mathrm{~m}$ \\
\hline Perturbation frequency & $0.5 \mathrm{~Hz}$ \\
\hline
\end{tabular}

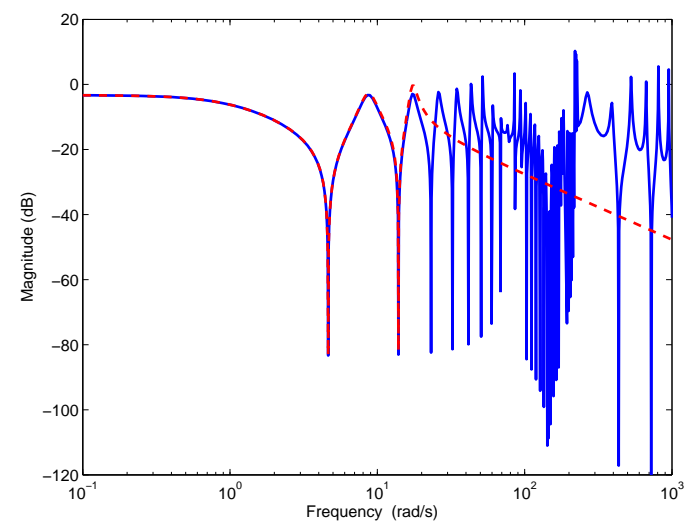

Fig. 3. Bode diagram comparison between the high order and the reduced order models for the transfer function from the perturbation in the draft tube to the mechanical torque.

The hydraulic model obtained has 241 states hence we need a reduced-order model more suitable to control design. We carry out a frequency analysis of the transfer functions from the guide vane opening $\alpha$ and the perturbation $h_{w}$ to the mechanical torque $T_{m}$. For this purpose numerical values of a physical system are needed, and as an example, the data of a real project developed by Alstom are considered. We also have used the laboratory and on-site measurements at offdesign operation of the turbine necessary to characterize the perturbation. The main specifications of the physical system can be found in Table III.

For the model reduction, we make the assumption that the actuator bandwidth is limited to $5 \mathrm{~Hz}$, and the pressure perturbation induced by the partial load vortex rope has a frequency, $\omega_{h}=0.5 \mathrm{~Hz}$ in (1). Hence, we keep the poles and zeros of the system in this frequency range. Fig. 3 shows the Bode diagram comparison for the transfer function between the perturbation input $h_{w}$ and the mechanical torque $T_{m}$.

The resulting state-space hydraulic model of order 7 is represented by

$$
\begin{aligned}
& \dot{X}_{h}=A_{h} X_{h}+B_{h} \alpha+B_{h e} \omega_{r}+B_{h w} h_{w} \\
& Y_{h}=T_{m}=C_{h} X_{h}+D_{h}\left(\begin{array}{c}
\alpha \\
\omega_{r}
\end{array}\right),
\end{aligned}
$$

where $X_{h}$ is the state vector, $\alpha$ the guide vane opening (control input), $\omega_{r}$ the rotational frequency, $h_{w}$ the head perturbation from (1) and $Y_{h}$ the output. All these variables are deviations around an operating point. 
The matrix entries of $A_{h}, B_{h e}, B_{h w}, C_{h}$ and $D_{h}$ can be found in Appendix I.

\section{Electro-Mechanical Subsystem Mathematical Model}

We model the generator and the electrical network as the well known SMIB (Single Machine Infinite Bus) [3]. The third order nonlinear model is linearized around an operating point $\left(P_{t_{0}}, E_{t_{0}}, Q_{t_{0}}\right)$. The resulting linear state-space system is described by

$$
\begin{aligned}
\dot{X}_{e} & =A_{e} X_{e}+B_{e}\left(\begin{array}{c}
\Delta T_{m} \\
\Delta E_{f d}
\end{array}\right) \\
Y_{e} & =C_{e} X_{e} .
\end{aligned}
$$

where the algebraic expressions of the variables are defined as follows:

$$
\begin{array}{rlrl}
X_{e} & =\left(\begin{array}{c}
\Delta \omega_{r} \\
\Delta \delta \\
\Delta \Psi_{f d}
\end{array}\right), & Y_{e}=\left(\begin{array}{c}
\Delta \omega_{r} \\
\Delta P_{t} \\
\Delta Q_{t} \\
\Delta E_{t}
\end{array}\right), \\
A_{e} & =\left(\begin{array}{ccc}
a_{11} & a_{12} & a_{13} \\
a_{11} & 0 & 0 \\
0 & a_{12} & a_{13}
\end{array}\right), & B_{e}=\left(\begin{array}{cc}
b_{11} & 0 \\
0 & 0 \\
0 & b_{32}
\end{array}\right), \\
C_{e} & =\left(\begin{array}{ccc}
1 & 0 & 0 \\
0 & c_{22} & c_{23} \\
0 & c_{32} & c_{33} \\
0 & c_{42} & c_{43}
\end{array}\right) .
\end{array}
$$

The expressions of the $a_{i j}, b_{i j}$ and $c_{i j}$ constants and of the initial conditions can be found in Appendix II and the numerical values in Appendix I.

\section{Full Hydroelectric Mathematical Model}

We aggregate the two subsystems together to obtain a state-space model of a hydroelectric unit with a hydraulic perturbation in the draft tube:

$$
\begin{aligned}
& \dot{x}=A x+B u+B_{w} h_{w} \\
& y=C x,
\end{aligned}
$$

where the state vector $x$ is a concatenation of the hydraulic state vector $X_{h}$ and the electric state vector $X_{e}, y$ is the output vector, $u$ is the control input and $h_{w}$ is the hydraulic perturbation input.

$$
x=\left(\begin{array}{c}
X_{h} \\
X_{e}
\end{array}\right), \quad y=Y_{e}, \quad u=\left(\begin{array}{c}
\alpha \\
E_{f d}
\end{array}\right)
$$

The concatenated matrices are defined as follows:

$$
\begin{aligned}
& A=\left(\begin{array}{ccc|ccc} 
& & & 0 & 0 & 0 \\
& A_{h} & & B_{h e} & \vdots & \vdots \\
& & & 0 & 0 \\
\hline & b_{11} C_{h} & & & \\
0 & \cdots & 0 & & \tilde{A}_{e} & \\
0 & \cdots & 0 & &
\end{array}\right), \\
& \tilde{A}_{e}=A_{e}+\left(\begin{array}{ccc}
b_{11} D_{h_{2}} & 0 & 0 \\
0 & 0 & 0 \\
0 & 0 & 0
\end{array}\right) \text {, } \\
& B=\left(\begin{array}{c|c} 
& 0 \\
B_{h} & \vdots \\
& 0 \\
\hline b_{11} D_{h_{1}} & 0 \\
0 & 0 \\
0 & b_{32}
\end{array}\right), \quad C=\left(0_{4,7} \mid C_{e}\right) \text {. }
\end{aligned}
$$

\section{MODEL SIMULATION}

We now compare our developed 10th order model to the full nonlinear model through simulation in closed-loop with the system subject to a pressure perturbation in the draft tube represented by (1) with $A_{h}=0.055$ p.u. and $\omega_{h}=\pi$ which corresponds to a perturbation frequency of $0.5 \mathrm{~Hz}$. This comparison is done with the classical controllers designed to have both good tracking and regulation performance on the active power for the turbine and the voltage for the generator. The full nonlinear model is implemented in the software package Simsen [13], which has been validated with on-site measurements to simulate the hydraulic subsystem. The turbine is modeled with the nonlinear hill charts (2) and the hydraulic subsystem is modeled with a high number of pipe elements in each pipe using (7). The generator mathematical model is the 7-th order nonlinear model with damper windings along with the second order network model [3] with $X_{E}=0.3$. The resulting model has an order 130 . The same controller topologies are used for both models, the results are displayed in Fig. 4 for the active power and the voltage. The deviations of the reduced model from the full model are displayed in Fig. 5. Wee see that our reduced model is close to the full model in this case.

\section{CONCLUSION}

We developed a 10th order model of a hydroelectric unit subject to hydraulic perturbations in the draft tube, for example pressure oscillations induced by partial or full load vortex rope. This model captures the dynamics of the water-hammer effet (travelling waves) in the pipes, we used the finite element method in this purpose and the model was reduced by keeping poles and zeros in the frequency region of interest for the control loop. Finally, simulation results were presented of our designed model compared to the full nonlinear model when the system is subject to pressure perturbation in the draft tube. The details of a control algorithm designed with this model will be published in a forthcoming paper. 


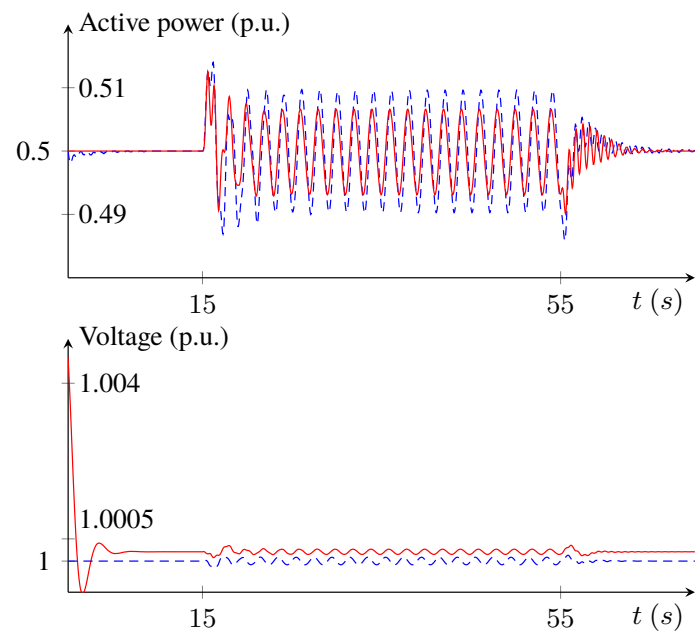

Fig. 4. Comparison of 10 th order model (-) with the 130th order model (-) in closed-loop whith the system subject to a sinusoidal head perturbation of amplitude $4 \mathrm{~m}$ and frequency $0.5 \mathrm{~Hz}$ from $15 \mathrm{~s}$ to $55 \mathrm{~s}$.

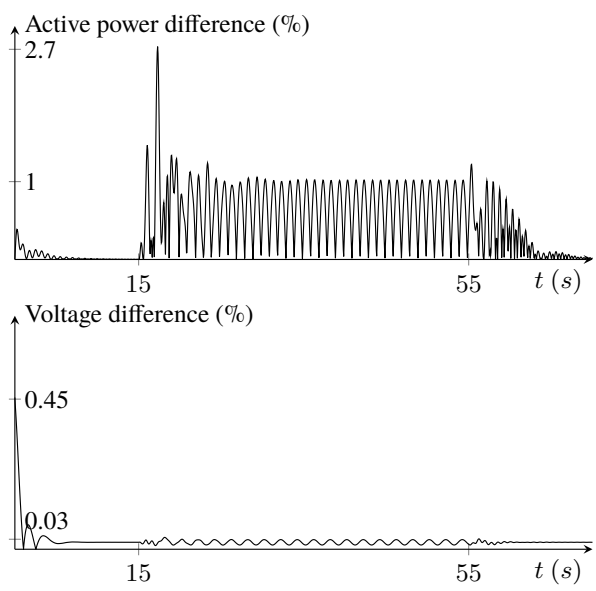

Fig. 5. Deviation of the 10th order model from the 130th order model in closed-loop whith the system subject to a sinusoidal head perturbation of amplitude $4 \mathrm{~m}$ and frequency $0.5 \mathrm{~Hz}$ from $15 \mathrm{~s}$ to $55 \mathrm{~s}$.

\section{APPENDIX I \\ Numerical VALUES}

\section{A. Hydraulic Subsystem}

The hydraulic subsystem is linearized around the operating point $T_{m_{0}}=0.5$ p.u. with the parameters given in Table IV. The numerical values of the matrices of (10) are given here:

TABLE IV

HYDRAULiC NUMERICAL VALUES

\begin{tabular}{c|cc} 
Penstock Pipe 1 \& 2 & \multicolumn{2}{|c}{ Turbine } \\
\hline$L_{1}=400 \mathrm{~m} \quad L_{2}=13 \mathrm{~m}$ & $\alpha_{1}=0.39$ & $\beta_{1}=1.11$ \\
$N_{b}=40$ & $\alpha_{2}=1.34$ & $\beta_{2}=1.36$ \\
$D=7.90 \mathrm{~m}$ & $\alpha_{3}=-1.64$ & $\beta_{3}=-11.29$ \\
$f=10^{-2}$ & \multicolumn{2}{|c|}{$\gamma_{\max }=35.5^{\circ}$}
\end{tabular}

$$
\begin{aligned}
& A_{h}= \\
& \left(\begin{array}{ccccccc}
-0.81 & 13.1 & -7.4 & 0.52 & 1.1 & 5.1 & 5.8 \\
-12.9 & -1.0 & 3.1 & 1.8 & 5.2 & 4.0 & -1.7 \\
7.6 & -3.4 & -0.59 & 22.1 & 5.4 & 6.1 & 2.1 \\
-0.7 & -1.8 & -22.2 & -0.77 & 4.1 & -8.4 & -2.8 \\
-0.94 & -5.2 & -5.0 & -3.8 & -1.2 & 4.6 & -4.3 \\
-5.3 & -4.2 & -6.1 & 8.6 & -4.9 & -1.1 & -1.2 \\
-6.2 & 2.0 & -2.9 & 3.0 & 4.4 & 1.1 & -1.1
\end{array}\right) \\
& B_{h}=\left(\begin{array}{c}
-0.33 \\
-0.99 \\
0.18 \\
-1.6 \\
1.2 \\
-1.0 \\
-2.8
\end{array}\right) \quad B_{h e}=\left(\begin{array}{c}
0.027 \\
1.1 \\
-0.68 \\
1.9 \\
-1.1 \\
0.92 \\
2.9
\end{array}\right) \\
& B_{h w}=\left(\begin{array}{c}
-0.15 \\
-0.14 \\
-0.10 \\
0.040 \\
-0.50 \\
0.10 \\
0.40
\end{array}\right) \\
& C_{h}=\left(\begin{array}{lllllll}
1.4 & -2.0 & 1.9 & -3.5 & 2.8 & -0.93 & -5.2
\end{array}\right) \\
& D_{h}=\left(\begin{array}{ll}
-2.3 & -4.6
\end{array}\right)
\end{aligned}
$$

\section{B. Electrical Subsystem}

The electrical subsystem is linearized around the operating point $P_{t_{0}}=0.5$ p.u., $E_{t_{0}}=1$ p.u. and $Q_{t_{0}}=$ 0 p.u. which is equivalent to considering the bus voltage $E_{B_{0}}=1$ p.u.. The matrices of (11) are expressed below.

$$
\begin{aligned}
& A_{e}=\left(\begin{array}{rrr}
0 & -0.18 & -0.11 \\
314 & 0 & 0 \\
0 & -0.073 & -0.27
\end{array}\right) \quad B_{e}=\left(\begin{array}{cc}
0.16 & 0 \\
0 & 0 \\
0 & 0.13
\end{array}\right) \\
& C_{e}=\left(\begin{array}{rrr}
1 & -0.073 & -0.27 \\
0 & 1.10 & 0.69 \\
0 & 0.14 & 1.39 \\
0 & -0.0044 & 0.40
\end{array}\right)
\end{aligned}
$$

With the synchronous machine parameter numerical values detailed in Table V.

TABLE V

ELECTRICAL NUMERICAL VALUES

\begin{tabular}{rlrl}
\hline$X_{d s}$ & $=0.971$ & $L_{l}$ & $=0.175$ \\
$X_{q s}$ & $=0.682$ & $R_{a}$ & $=2.58 \times 10^{-3}$ \\
$L_{a d u}$ & $=0.918$ & $X_{d s}^{\prime}$ & $=0.277$ \\
$L_{a d s}$ & $=0.796$ & $L_{a q s}$ & $=0.507$ \\
$L_{f d}$ & $=0.155$ & $R_{f d}$ & $=3.92 \times 10^{-4}$ \\
$X_{E}$ & $=0.3$ & $R_{E}$ & $=0.03$ \\
\hline
\end{tabular}

\section{APPENDIX II}

\section{ELECTRO-MECHANICAL MODEL}

In this section, we define the matrices of the electromechanical state-space model as well as the expressions to calculate the initial conditions of the machine. Table II is the nomenclature of all electro-mechanical variables.

\section{A. Matrix Constants}

We develop here the expressions for the matrix constants of (11), [3].

$$
a_{11}=-\frac{K_{D}}{2 H} \quad a_{12}=-\frac{K_{1}}{2 H} \quad a_{13}=-\frac{K_{2}}{2 H}
$$




$$
\begin{aligned}
& a_{21}=\omega_{0}=2 \pi f_{0} \quad a_{32}=-\frac{\omega_{0} R_{f d}}{L_{f d}} m_{1} L_{a d s}^{\prime} \\
& a_{33}=-\frac{\omega_{0} R_{f d}}{L_{f d}}\left[1-\frac{L_{a d s}^{\prime}}{L_{f d}}+m_{2} L_{a d s}^{\prime}\right] \\
& b_{11}=\frac{1}{2 H} \quad b_{32}=\frac{\omega_{0} R_{f d}}{L_{a d u}} \\
& c_{22}=n_{1}\left(\Psi_{a d 0}+L_{a q s} i_{d 0}-R_{a} i_{q 0}\right) \\
& -m_{1}\left(\Psi_{a q 0}+L_{a d s}^{\prime} i_{q 0}+R_{a} i_{d 0}\right) \\
& c_{23}=n_{2}\left(\Psi_{a d 0}+L_{a q s} i_{d 0}-R_{a} i_{q 0}\right) \\
& -m_{2}\left(\Psi_{a q 0}+L_{a d s}^{\prime} i_{q 0}+R_{a} i_{d 0}\right)+\frac{L_{a d s}^{\prime}}{L_{f d}} i_{q 0} \\
& c_{32}=m_{1}\left(\Psi_{a d 0}-\left(L_{l}+L_{a d s}^{\prime}\right) i_{d 0}\right) \\
& +n_{1}\left(\Psi_{a q 0}-\left(L_{l}+L_{a q s}\right) i_{q 0}\right) \\
& c_{33}=m_{2}\left(\Psi_{a d 0}-\left(L_{l}+L_{a d s}^{\prime}\right) i_{d 0}\right) \\
& +n_{2}\left(\Psi_{a q 0}-\left(L_{l}+L_{a q s}\right) i_{q 0}\right)+\frac{L_{a d s}^{\prime}}{L_{f d}} i_{d 0} \\
& c_{42}=\frac{e_{d 0}}{E_{t 0}}\left(-R_{a} m_{1}+L_{l} n_{1}+L_{a q s} n_{1}\right) \\
& +\frac{e_{q 0}}{E_{t 0}}\left(-R_{a} n_{1}+L_{l} m_{1}+L_{a d s}^{\prime} m_{1}\right) \\
& c_{43}=\frac{e_{d 0}}{E_{t 0}}\left(-R_{a} m_{2}+L_{l} n_{2}+L_{a q s} n_{2}\right) \\
& +\frac{e_{q 0}}{E_{t 0}}\left(-R_{a} n_{2}+L_{l} m_{2}+L_{a d s}^{\prime}\left[\frac{1}{L_{f d}}-m_{2}\right]\right) \\
& K_{1}=n_{1}\left(\Psi_{a d 0}+L_{a q s} i_{d 0}\right)-m_{1}\left(\Psi_{a q 0}+L_{a d s}^{\prime} i_{q 0}\right) \\
& K_{2}=n_{2}\left(\Psi_{a d 0}+L_{a q s} i_{d 0}\right)-m_{2}\left(\Psi_{a q 0}+L_{a d s}^{\prime} i_{q 0}\right) \\
& +\frac{L_{a d s}^{\prime}}{L_{f d}} i_{q 0} \\
& m_{1}=\frac{E_{B}\left(X_{T_{q}} \sin \delta_{0}-R_{T} \cos \delta_{0}\right)}{D} \\
& n_{1}=\frac{E_{B}\left(R_{T} \sin \delta_{0}+X_{T_{d}} \cos \delta_{0}\right)}{D} \\
& m_{2}=\frac{X_{T_{q}}}{D} \frac{L_{a d s}}{\left(L_{a d s}+L_{f d}\right)} \quad n_{2}=\frac{R_{T}}{D} \frac{L_{a d s}}{\left(L_{a d s}+L_{f d}\right)} \\
& X_{T_{q}}=X_{E}+\left(L_{a q s}+L_{l}\right)=X_{E}+X_{q s} \\
& X_{T_{d}}=X_{E}+\left(L_{a d s}^{\prime}+L_{l}\right)=X_{E}+X_{d s}^{\prime} \\
& D=R_{T}^{2}+X_{T_{q}} X_{T_{d}} \quad R_{T}=R_{a}+R_{E} \\
& L_{a d s}^{\prime}=\frac{1}{\frac{1}{L_{a d s}}+\frac{1}{L_{f d}}}
\end{aligned}
$$

\section{B. Initial Conditions}

$P_{t 0}, Q_{t 0}$ and $E_{t 0}$ at the operating point are fixed and the following expressions are used to compute the initial conditions [3].

$$
\begin{aligned}
I_{t} & =\frac{\sqrt{P_{t}^{2}+Q_{t}^{2}}}{E_{t}} \quad \Phi=\cos ^{-1}\left(\frac{P_{t}}{E_{t} I_{t}}\right) \\
\delta_{i} & =\tan ^{-1}\left(\frac{X_{q s} I_{t} \cos \Phi-R_{a} I_{t} \sin \Phi}{E_{t}+R_{a} I_{t} \cos \Phi+X_{q s} I_{t} \sin \Phi}\right)
\end{aligned}
$$

$$
\begin{aligned}
e_{d_{0}} & =E_{t} \sin \delta_{i} & e_{q_{0}} & =E_{t} \cos \delta_{i} \\
i_{d_{0}} & =I_{t} \sin \left(\delta_{i}+\Phi\right) & & i_{q_{0}}=I_{t} \cos \left(\delta_{i}+\Phi\right) \\
E_{B d_{0}} & =e_{d_{0}}-R_{E} i_{d_{0}}+X_{E} i_{q_{0}} & & \\
E_{B q_{0}} & =e_{q_{0}}-R_{E} i_{q_{0}}-X_{E} i_{d_{0}} & & \\
E_{B_{0}} & =\sqrt{E_{B d_{0}}^{2}+E_{B q_{0}}^{2}} & \delta_{0} & =\tan ^{-1}\left(\frac{E_{B d_{0}}}{E_{B q_{0}}}\right) \\
i_{f d_{0}} & =\frac{e_{q_{0}}+R_{a} i_{q_{0}}+L_{d s} i_{d_{0}}}{L_{a d s}} & E_{f d_{0}} & =L_{a d u} i_{f d_{0}} \\
\Psi_{a d_{0}} & =L_{a d s}\left(-i_{d_{0}}+i_{f d_{0}}\right) & \Psi_{a q_{0}} & =-L_{a q s} i_{q_{0}}
\end{aligned}
$$$$
\text { ACKNOWLEDGMENT }
$$

The first author is funded by CIFRE Ph.D. grant $n$. 2013/1187.

The authors would like to thank Thomas de Colombel and Josselin Guillozet for the enlightening discussions on turbine partial loading pressure oscillations.

\section{REFERENCES}

[1] C. Nicolet, J.-J. Herou, B. Greiveldinger, P. Allenbach, J.-J. Simond, and F. Avellan, "Methodology for risk assessment of part load resonance in Francis turbine power plant," in Proceedings IAHR Int. Meeting of WG on Cavitation and Dynamic Problems in Hydraulic Machinery and Systems, June 2006.

[2] M. H. Chaudhry, Applied Hydraulic Transients. Springer-Verlag New York Inc., 2014.

[3] P. Kundur, Power System Stability and Control. New York: McGrawHill, 1994.

[4] "Hydraulic turbine and turbine control models for system dynamic studies," Power Systems, IEEE Transactions on, vol. 7, no. 1, February 1992.

[5] G. Orelind, L. Wozniak, J. Medanic, and T. Whittemore, "Optimal PID gain schedule for hydrogenerators - design and application," Energy Conversion, IEEE Transactions on, vol. 4, no. 3, September 1989.

[6] J. Jiang, "Design of an optimal robust governor for hydraulic turbine generating units," Energy Conversion, IEEE Transactions on, vol. 10, no. 1, March 1995.

[7] O. Malik and Y. Zeng, "Design of a robust adaptive controller for a water turbine governing system," Energy Conversion, IEEE Transactions on, vol. 10, no. 2, June 1995.

[8] G. Muoz-Hernandez and D. Jones, "MIMO generalized predictive control for a hydroelectric power station," Energy Conversion, IEEE Transactions on, vol. 21, no. 4, December 2006.

[9] S. Alligne, C. Nicolet, Y. Tsujimoto, and F. Avellan, "Cavitation surge modelling in francis turbine draft tube," Journal of Hydraulic Research, pp. 1-13, 2014.

[10] P. K. Doerfler, "Evaluating 1D models for vortex-induced pulsation in Francis turbines," in 3rd IAHR International Meeting of the Workgroup on Caviation and Dynamic Problemes in Hydraulic Machinery and Systems, October 2009.

[11] A. Padoan, B. Kawkabani, A. Schwery, C. Ramirez, C. Nicolet, J.-J. Simond, and F. Avellan, "Dynamical behavior comparison between variable speed and synchronous machines with PSS," Power Systems, IEEE Transactions on, vol. 25, no. 3, August 2010.

[12] J. Mallik, "Reducing the effect of vortex rope oscillation on hydro units by power system stabilizer designed using genetic algorithm," in India Conference (INDICON), 2011 Annual IEEE, December 2011, pp. $1-4$.

[13] C. Nicolet, F. Avellan, P. Allenbach, A. Sapin, and J. Simond, "New tool for the simulation of transient phenomena in Francis turbine power plants," in Proceedings of the Hydraulic Machinery and Systems 21st IAHR Symposium, September 2002. 